\title{
Tag SNP Selection Based on Multivariate Linear Regression
}

\author{
Jingwu He ${ }^{\star}$ and Alex Zelikovsky ${ }^{\star \star}$ \\ Department of Computer Science, Georgia State University, GA 30303, Atlanta \\ $\{$ jingwu, alexz $\}$ @cs.gsu.edu
}

\begin{abstract}
The search for the association between complex diseases and single nucleotide polymorphisms (SNPs) or haplotypes has been recently received great attention. For these studies, it is essential to use a small subset of informative SNPs (tag SNPs) accurately representing the rest of the SNPs. Tagging can achieve budget savings by genotyping only a limited number of SNPs and computationally inferring all other SNPs and compaction of extremely long SNP sequences (obtained, e.g., from Affimetrix Map Array) for further fine genotype analysis. Tagging should first choose tags from the SNPs under consideration and then knowing the values of chosen tag SNPs predict (or statistically cover) the non-tag SNPs. In this paper we propose a new SNP prediction method based on rounding of multivariate linear regression (MLR) analysis in sigmarestricted coding. When predicting a non-tag SNP, the MLR method accumulates information about all tag SNPs resulting in significantly higher prediction accuracy with the same number of tags than for the previously known tagging methods. We also show that the tag selection strongly depends on how the chosen tags will be used - advantage of one tag set over another can only be considered with respect to a certain prediction method. Two simple universal tag selection methods have been applied: a (faster) stepwise and a (slower) local-minimization tag selection algorithms. An extensive experimental study on various datasets including 6 regions from HapMap shows that the MLR prediction combined with stepwise tag selection uses significantly fewer tags (e.g., up to two times less tags to reach $90 \%$ prediction accuracy) than the state-ofart methods of Halperin et al. 8 for genotypes and Halldorsson et al. [7] for haplotypes, respectively. Our stepwise tagging matches the quality of while being faster than STAMPA 8]. The code is publicly available at http://alla.cs.gsu.edu/ software.
\end{abstract}

\section{Introduction}

The search for the association between complex diseases and single nucleotide polymorphisms (SNPs) or haplotypes has been recently received great attention.

\footnotetext{
* Partially supported by GSU Molecular Basis of Disease Fellowship.

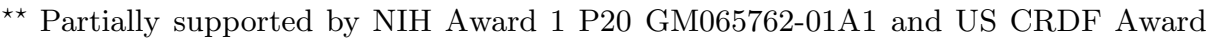
\#MOM2-3049-CS-03.
} 
For these studies, it is essential to use a small subset of informative SNPs (tag SNPs) accurately representing the rest of the SNPs. Tagging can achieve budget savings by genotyping only a limited number of SNPs and computationally inferring all other SNPs and compaction of extremely long SNP sequences (obtained, e.g., from Affimetrix Map Array) for further fine genotype analysis.

Tagging methods have been previously explored in statistical and pattern recognition community as well as optimization community. In statistics, tags are required to statistically cover individual (non-tagged) SNPs or haplotypes (sets of SNPs), where the quality of statistical covering is usually measured by correlation, e.g., find minimum number of tags such that for any non-tag SNP there exists a highly correlated (squared correlation $R^{2}>.8$ ) tag SNP [2, 4]. In the optimization community, the number of tags is usually minimized subject to upper bounds on prediction error measured in leave-one-out cross-validation experiments [7, 8].

The generic tagging problem can be formulated as follows: Given a sample $S$ of a population $P$ of individuals (either haplotypes or genotypes) on $m$ SNPs, find positions of $k(k<m)$ tag SNPs such that one can predict (or statistically cover) an entire individual (haplotype or genotype) from its restriction onto the $k$ tag SNPs. This tagging problem formulation implicitly relies on a certain prediction or statistical covering method. The corresponding SNP prediction problem is formulated as follows: Given the values of $k$ tags of the individual $x$ with unknown SNP $s$ and $n$ individuals with $k$ tag SNP and known value of SNP $s$, find the value of $s$ in $x$. Respectively, each non-tag SNP must be statistically covered by a tag SNP or multiple tag SNPs as proposed below.

In this paper we propose a new SNP prediction based on rounding of multivariate linear regression (MLR) analysis in sigma-restricted coding. The MLR method predicts the non-tag SNP $s$ so that the predicted $s$ is the closest to its projection on the span of vectors corresponding to tag SNPs. Respectively, the MLR method statistically covers $s$ by its projection. The MLR method accumulates information about all tag SNPs resulting in significantly higher prediction accuracy with the same number of tags. In contrast, the previous SNP prediction methods rely either on a single SNP (see, e.g., 2]), a pair of tag SNPs 8, or small number of tag SNPs from the block with limited haplotype diversity [14]. Our previous linear-algebraic tag selection and SNP prediction [9, 10] picks linear independent tag SNPs but cannot easily handle bounds on prediction error or number of tags. We show that the tag selection strongly depends on how the chosen tags will be used - advantage of one tag set over another can only be considered with respect to a certain prediction method. We suggest two simple universal methods for tag selection based on known prediction method: a (faster) stepwise and a (slower) local-minimization tag selection algorithms.

An extensive experimental study on various datasets including 6 regions from HapMap [1] shows that the MLR prediction combined with stepwise tag selection uses significantly fewer tags (e.g., up to two times less tags to reach $90 \%$ prediction accuracy) than the state-of-art methods of Halperin et al. 8] for genotypes and Halldorsson et al. [7] for haplotypes, respectively. Our stepwise tagging matches 
the quality of while being faster than STAMPA [8. When applied to statistical covering, our method also uses fewer tags than the state-of-the-art method of Carlson et al. 2] especially when high correlation $\left(r^{2}>.8\right)$ is required.

The rest of the paper is organized as follows: Section 2 describes our MLR SNP prediction algorithm. Section 3 shows how to separate tag selection problem from SNP prediction or statistical covering and describes the general approach for tag selection based on the way how tags are used. Section 4 presents an experimental results and discussions.

\section{The MLR SNP Prediction Algorithm}

Usually, a genotype is represented by a vector with coordinates 0,1 , or 2 , where 0 represents the homozygous site with major allele, 1 represents the homozygous site with minor allele, and 2 represents the heterozygous site. Respectively, each haplotype's coordinate is 0 or 1 , where 0 represents the major allele and 1 represents the minor allele. The sample population $S$ together with the tag-restricted individual $x$ are represented as a matrix $M$. The matrix $M$ has $n+1$ rows corresponding to $n$ sample individuals and the individual $x$ and $k+1$ columns corresponding to $k$ tag SNPs and a single non-tag SNP $s$. All values in $M$ are known except the value of $s$ in $x$. In case of haplotypes, there are only two possible resolutions of $s$, namely, $s_{0}$ and $s_{1}$ with the unknown SNP value equal to 0 or 1 , respectively. For genotypes, there are 3 possible resolutions $s_{0}, s_{1}$, and $s_{2}$ corresponding to SNP values 0,1 , or 2 , respectively. The SNP prediction method should chose correct resolution of $s$.

The proposed MLR SNP prediction method considers all possible resolutions of $s$ together with the set of tag SNPs $T$ as the vectors in $(n+1)$-dimensional Euclidean space. It assumes that the most probable resolution of $s$ should be the "closest" to $T$. The distance between resolution of $s$ and $T$ is measured between $s$ and its projection on the vector space $\operatorname{span}(T)$, the span of the set of tag SNPs $T$ (see Figure 1).



Fig. 1. MLR SNP Prediction Algorithm. Three possible resolutions $s_{0}, s_{1}$, and $s_{2}$ of $s$ are projected on the span of tag SNPs (a dark plane). The unknown SNP value is predicted 1 since the distance between $s_{1}$ and its projection $s_{1}^{T}$ is the shorter than for $s_{0}$ and $s_{2}$. 
Computationally, the distance between a resolution $s_{i}$ and $T$ is measured as $\operatorname{dist}\left(T, s_{i}\right)=\left|T \cdot\left(T^{t} \cdot T\right)^{-1} \cdot T^{t} \cdot s_{i}-s_{i}\right|$. The runtime of the MLR SNP prediction algorithm is $O\left(k n^{2}\right)$. In general, there are $m-k$ non-tag SNPs in each individual but the matrix $T \cdot\left(T^{t} \cdot T\right)^{-1} \cdot T^{t}$ is the same for all these non-tag SNPs and should be computed only once. Thus, the total runtime for predicting a complete individual is $O(k n(n+m))$.

Instead of $\{0,1,2\}$ notation, we use sigma-encoding $\{-1,1,0\}$ of SNP values proposed in [10]: 0's are replaced by -1 's and 2's are replaced with 0's. Our experiments show that MLR with $\{-1,0,1\}$ notations needs up to $30 \%$ less tags than with the original $\{0,2,1\}$ notations for reaching same prediction accuracy.

\section{$3 \quad$ Tag SNP Selection Methods}

Assuming self-similarity of data, one can expect that an algorithm predicting with high accuracy SNPs of an unknown individual will also predict with high accuracy SNPs of the sampled individual. Then, we expect that the better prediction algorithm will have fewer errors when predicting SNPs in the sample $S$. This expectation allows us to find tags using prediction algorithm as follows: We can check each $k$-tuple of tags and choose the $k$-tuple with the minimal number of errors in predicting the non-tag SNPs in the sampled individuals. Even though the sample elements are completely typed, prediction algorithms can make still errors because the number of SNPs may be not sufficient to distinguish any two sampled individuals. Thus, tag SNP selection based on prediction is reduced to the following problem:

Tag SNP Selection for Prediction. Given a prediction algorithm $A_{k}$ and a sample $S$, find $k$ tags such that the prediction error e of $A_{k}$ averaged over all SNPs in $S$ (including tags) is minimized.

Similarly, a statistical covering criteria has as an input a set of $k$ column-tags $\left(t_{1}, \cdots, t_{k}\right)$ and a single SNP $s$ on the sample $S . A_{k}$ checks if the set of tags has a statistically significant correlation with $s$. For example, in Carlson et al. 2] $A_{k}$ checks if the $R^{2}$ between $s$ and $t_{i}$ 's is higher than a certain threshold (e.g., 0.8). A similar criteria is defined in Stram et al. 13. In this paper, we suggest to compute $A_{k}$ as a correlation between $s$ and $s^{i}$ predicted using MLR algorithm. The statistical covering version of the tag SNP selection can be formulated as follows:

Tag SNP Selection for Statistical covering. Given a statistical covering criteria $A_{k}$ and a sample $S$, find $k$ tags such that the number of statistically covered SNPs (including tags) according to $A_{k}$ is maximized.

In general, these problems are computationally difficult and the runtime of an exact algorithm may become prohibitively slow. Below we propose two universal heuristics which can be applied to an arbitrary prediction algorithm or statistical covering criteria $A_{k}$.

The Stepwise Tagging algorithm (STA) starts with the best tag $t_{0}$, i.e., tag that minimizes error when predicting with $A_{k}$ all other tags. Then STA finds 
such tag $t_{1}$ which would be the best extension of $\left\{t_{0}\right\}$ and continue adding best tags until reaching the set of tags of the given size $k$. STA produces hereditary set of tags, i.e., the chosen $k$ tags contain the chosen $k-1$ tags. This hereditary property may be useful in case if the set of tags can be extended. The runtime of STA is $O(k n m T)$, where $T$ is the runtime of the prediction algorithm. Note that for statistical covering, STA is equivalent to the greedy algorithm used by Carlson et al. 2].

The Local-Minimization Tag Selection algorithm (LMT) is more accurately searching for a better set of tags among much larger possibilities. LMT starts with the $k$ tags produced by STA and then iteratively replaces each single tag with the best possible choice while not changing other tags. Such replacements will be continued until no significant improvement in the prediction quality (i.e., by more than given amount of $\epsilon \%)$ can be achieved. The runtime of LMT is $O\left(k n m T \epsilon^{-1}\right)$ since the number of iterations cannot exceed $\frac{100}{\epsilon}$.

\section{Experimental Results}

We first list the test datasets, then compare the MLR prediction method combined with STA and LMT. Next comparison of prediction accuracy and statistical covering is given as well comparison of MLR with Idselect. Finally, we give the comparison results of MLR with the state-of-art genotype tagging of STAMPA [8], and haplotype tagging of Haldorsson et al. 7] and He et al. [10].

The following datasets are used to measure the quality of our algorithms. Currently, our algorithms cannot tolerate missing data. Following Halperin et al. 8], we use GERBIL [6] to phase the genotypes and then combine the resulting two haplotypes to recover any missing data.

Three ENCODE regions. Three regions (ENm013, ENr112, ENr113) from 30 CEPH family trios obtained from HapMap ENCODE Project [1]. The number of SNPs genotyped in each region is 361,412 and 515 respectively.

Two gene regions. Two gene regions STEAP and TRPM8 from 30 CEPH family trios obtained from HapMap [1. The number of SNPs genotyped in each gene region is 23 and 102 SNPs.

Chromosome 5q31. The data set collected by Daly et al. 5] derived from the 616 kilobase region of human Chromosome 5q31 from 129 family trios.

LPL \& Chromosome 21. The Clark et al. 3] data set consists of the haplotypes of 71 individuals typed over 88 SNPs in the human lipoprotein lipase (LPL) gene. The Chromosome 21 data set consists the first 1,000 of 24,047 SNPs typed over 20 haploid copies of human Chromosome 21 [1].

We apply leave-one-out cross-validation to evaluate the quality of the solution given by the tag SNP selection and prediction methods. One by one, each individual is removed from the sample. Then, tag SNPs are selected using only the remaining individuals. The "left out" individual is reconstructed based on its tag SNPs and the remaining individuals in the sample. The prediction accuracy is percent of correctly predicted SNPs in all individuals. 
Table 1. The number of tag SNPs sufficient for MLR/STA to reach prediction accuracy between $80 \%$ and $99 \%$. The ${ }^{*}$ 's indicate cases when MLR/LMT needs one tag less than MLR/STA.

\begin{tabular}{c|ccccccccccccc}
\hline & \multicolumn{10}{|c}{ prediction accuracy, \% } \\
Datasets & 80 & 85 & 90 & 91 & 92 & 93 & 94 & 95 & 96 & 97 & 98 & 99 \\
\hline ENm013 & 2 & 3 & 6 & 6 & 7 & 8 & 9 & 9 & 11 & 15 & 22 & 254 \\
ENr112 & 6 & 9 & 14 & 16 & 18 & 20 & 24 & 33 & 63 & 95 & 126 & 187 \\
ENr113 & 4 & 5 & 10 & 11 & 13 & 15 & 18 & 40 & 55 & 80 & 104 & 200 \\
STEAP & 1 & 1 & 1 & 2 & 2 & 2 & 2 & 2 & $3^{*}$ & 3 & $4^{*}$ & 4 \\
TRPM8 & 1 & 2 & 4 & 5 & 5 & 6 & 7 & 8 & 10 & 15 & 15 & 24 \\
5q31 & 1 & 2 & 5 & 7 & 7 & 9 & 13 & 16 & 21 & 31 & 41 & 55 \\
\hline
\end{tabular}

Table 2. The number of tag SNPs used by MLR/STA and STAMPA to achieve $80 \%$ and $90 \%$ prediction accuracy in leave-one-out tests for 6 datasets

\begin{tabular}{c|c|c|c|c|c|c|c}
\hline Acc. & Algorithm & ENm013 & ENr112 & ENr113 & STEAP & TRPM8 & $5 q 31$ \\
\hline $80 \%$ & MLR & 2 & 6 & 4 & 1 & 1 & 1 \\
& STAMPA & 5 & 9 & 11 & 2 & 3 & 2 \\
\hline $90 \%$ & MLR & 6 & 14 & 10 & 1 & 4 & 5 \\
& STAMPA & 12 & 17 & 18 & 2 & 6 & 6 \\
\hline
\end{tabular}

Table 1 presents the results of leave-one-out experiments on the 6 genotype datasets. STA combined with MLR (MLR/STA) has almost the same quality as LMT combined with MLR (MLR/LMT) while being much faster. For example, when we perform the test on TRPM8 dataset, MLR/STA needs 4 seconds to reach $95 \%$ prediction accuracy while MLR/LMT needs 51 seconds 1

Table 2 compares the prediction accuracy of MLR/STA and STAMPA (Halperin et al. 8]). MLR/STA requires fewer tag SNPs and faster than STAMPA. For example, for TRPM8 data, MLR/STA in 6 seconds selects 12 tags to reach $96.6 \%$ accuracy, while STAMPA in 34 seconds selects 12 tags to reach only $94.2 \%$ accuracy. Since the MLR prediction algorithm can be applied to haplotypes, we compare it with the methods of Halldorson et al. [7] and $\mathrm{He}$ et al. [10] in leave-one-out tests on the LPL \& Chromosome 21 datasets. Figure 2 shows that the MLR/STA considerably improves over the both methods.

For maximizing statistical covering, each (non-tag) SNP-column $s$ is predicted with the MLR prediction algorithm. We say that the SNP-column $s$ is counted as statistically covered if squared correlation $R^{2}$ between the predicted SNPcolumn $s^{\prime}$ and given SNP-column $s$ is at least 0.8. In Table 3 the first two rows show the correlation of prediction accuracy and number of statistically covered SNPs. The third row shows that it is slightly better to use the correct objective (i.e., statistical covering) rather than prediction accuracy in order to maximize the number of statistically covered SNPs. Table 4 shows that MLR/STA uses on average 30\% fewer tags than IdSelect [2] for statistical covering all SNPs.

\footnotetext{
${ }^{1}$ All experiments are performed on a computer with Intel Pentium 4, 3.06Ghz processor and $2 \mathrm{~GB}$ of RAM.
} 

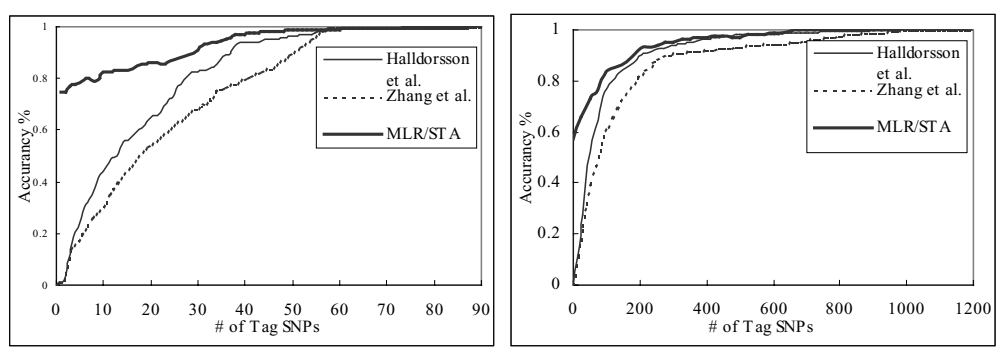

Fig. 2. Comparison of three haplotype prediction methods: Haldorsson et al. 7], He et al. 10, and MLR/STA. The x-axis shows the number of SNPs typed, and the yaxis shows the fraction of SNPs correctly imputed in a leave-one-out experiment. (A) Results from the LPL data set. (B) Results from the first 1000 SNPs of Chromosome 21 data set.

Table 3. The quality of MLR/STA on Daly et al. 5] data with two different tagging objectives over different number of tag SNPs

\begin{tabular}{c|c|ccccccc}
\hline objective & & \multicolumn{7}{|c}{ number of tag SNPs } \\
of tagging & & 0 & 1 & 2 & 4 & 6 & 8 & 10 \\
\hline SNP prediction & prediction accuracy, & 61.54 & 81.35 & 83.94 & 88.65 & 91.11 & 92.96 & 93.89 \\
SNP prediction & \# of SNPs covered & 0 & 10 & 16 & 36 & 47 & 53 & 59 \\
statistical covering & \# of SNPs covered & 0 & 11 & 24 & 38 & 50 & 54 & 61 \\
\hline
\end{tabular}

Table 4. The number of tag SNPs for statistical covering of all SNPs required by three methods: MLR/STA with prediction objective, MLR/STA with statistical covering objective, and IdSelect 2

\begin{tabular}{ccccccc}
\hline Algorithm & ENm013 & ENr112 & ENr113 & STEAP TRPM8 & 5q31 \\
\hline MLR (prediction) & 56 & 82 & 106 & 13 & 46 & 44 \\
MLR (statistical covering) & 51 & 71 & 85 & 11 & 41 & 41 \\
IdSelect & 71 & 122 & 132 & 16 & 53 & 51 \\
\hline
\end{tabular}

\section{Conclusions}

The paper gives a new SNP prediction method based on multivariate linear regression and suggests two universal tag selection methods. The MLR method combined with stepwise tag selection uses significantly fewer tags (e.g., up to two times less tags to reach $90 \%$ prediction accuracy) than the state-of-theart methods of Halperin et al. 8] for genotypes and Halldorsson et al. 7] for haplotypes, respectively. Our stepwise tagging matches the quality of while being faster than STAMPA [8]. When applied to statistical covering, our method also uses fewer tags than the state-of-the-art method of Carlson et al. [2] especially when high correlation $\left(R^{2}>.8\right)$ is required. 


\section{References}

1. http://www.hapmap.org

2. Carlson, C.S., Eberle, M.A., Rieder, M.J., Yi, Q., Kruglyak, L. and Nickerson, D.A. (2004) 'Selecting a maximally informative set of single-nucleotide polymorphisms for association analyses using linkage disequilibrium', American Journal of Human Genetics, Vol. 74, No. 1, pp. 106-120.

3. Clark, A., Weiss, K., Nickerson, D., Taylor, S., Buchanan, A., Stengard, J., Salomaa, V., Vartiainen, E., Perola, M., Boerwinkle, E. (1998) 'Haplotype structure and population genetic inferences from nucleotide-sequence variation in human lipoprotein lipase', American Journal of Human Genetics, Vol. 63, pp. 595-612.

4. Chapman, J.M., Cooper, J.D., Todd, J.A. and Clayton, D.G. (2003). 'Detecting disease associations due to linkage disequilibrium using haplotype tags: a class of tests and the determinants of statistical power', Human Heredity, Vol. 56, pp. $18-31$.

5. Daly, M., Rioux, J., Schaffner, S., Hudson, T. and Lander, E. (2001) 'High resolution haplotype structure in the human genome', Nature Genetics, Vol. 29, pp. 229-232.

6. Kimmel, G and Shamir R. (2004) 'GERBIL: Genotype resolution and block identification using likelihood', PNAS vol. 102, pp 158-162.

7. Halldorsson, B.V., Bafna, V., Lippert, R., Schwartz, R., de la Vega, F.M., Clark, A.G. and Istrail, S. (2004) 'Optimal haplotype block-free selection of tagging SNPs for genome-wide association studies', Genome Research Vol. 14, pp. 1633-1640.

8. Halperin, E., Kimmel, G. and Shamir, R. (2005) 'Tag SNP Selection in Genotype Data for Maximizing SNP Prediciton Accuracy', Bioinformatics 21:i195-i203;

9. He, J. and Zelikovsky, A. (2004) 'Linear Reduction Methods for Tag SNP Selection', Proceedings of the International Conference of the IEEE Engineering in Medicine and Biology, pp. 2840-2843.

10. He, J. and Zelikovsky, A. (2005) 'Linear Reduction Method for Predictive and Informative Tag SNP Selection', International Journal Bioinformtics Research and Applications, Vol. 3, pp. 249-260.

11. Patil, N., Berno, A., Hinds, D., Barrett, W., Doshi, J., Hacker, C., Kautzer, C., Lee, D., Marjoribanks, C., McDonough, D., Nguyen, B., Norris, M., Sheehan, J., Shen, N., Stern, D., Stokowski, R., Thomas, D., Trulson, M., Vyas, K., Frazer, K., Fodor, S. and Cox, D. (2001) 'Blocks of limited haplotype diversity revealed by high-resolution scanning of human chromosome', Science, Vol. 294, pp. 1719-1723.

12. StatSoft, Inc. (1999). Electronic Statistics Textbook. Tulsa, OK: StatSoft. WEB: http://www.statsoft.com/textbook/stathome.html.

13. Stram, D., Haiman, C., Hirschhorn, J., Altshuler, D., Kolonel, L., Henderson, B. and Pike, M. (2003). 'Choosing haplotype-tagging SNPs based on unphased genotype data using as preliminary sample of unrelated subjects with an example from the multiethnic cohort study', Human Heredity, Vol. 55, pp. 27-36.

14. Zhang, K., Qin, Z., Liu, J., Chen, T., Waterman, M., and Sun, F. (2004) 'Haplotype block partitioning and tag SNP selection using genotype data and their applications to association studies', Genome Research, Vol. 14, pp. 908-916. 